\title{
Heat Flow Birefringence in Liquids and Liquid Crystals
}

D. R. Baalss and S. Hess

Institut für Theoretische Physik, Glückstraße 6, Universität Erlangen-Nürnberg, D-8520 Erlangen, Germany

Z. Naturforsch. 40 a, 3-7 (1985); received October 25, 1984

The alignment of nonspherical particles is inferred from the solution of a Fokker-Planck equation where a thermal torque has been taken into account which is proportional to the second spatial derivative of the temperature field. A pretransitional enhancement of the effect is predicted for the isotropic phase of a liquid crystal. Two distinct physical mechanisms are considered in order to estimate the magnitude of the thermal torque. One of them is due to the pressure variation at constant density. For constant pressure, the torque is inferred from the collision term of an Enskog-Boltzmann equation generalized to (strongly) nonspherical particles. In both cases, the resulting heat flow birefringence is of measurable size.

Heat flow birefringence, i.e. a molecular alignment caused by the gradient of a heat flux or, equivalently, by the second spatial derivative of the temperature, has been predicted [1], calculated $[2-5]$, and measured $[6,7]$ for molecular gases. In contradistinction to flow birefringence where experiments with colloidal solutions [8,9] and liquids [10] preceded the theory $[1,11,12]$ and its experimental verification [13-15] for molecular gases by many decades, no measurements of the heat flow birefringence in the liquid phase are known to the authors. It has been pointed out before [3] that the phenomenological equation governing the heat flow birefringence in liquids should be analogous to the equation for gases despite the fact that the relevant alignment tensor has a different microscopic meaning in both cases and that the kinetic theory of [1-5] based on the Waldmann-Snider equation [16], of course, does not apply to liquids. For liquids and liquid crystals, one may develop a theory in the spirit of generalized irreversible thermodynamics by combining the ideas used for the viscous flow and flow birefringence in $[17,18]$ with those of Waldmann and Vestner [19] concerned with transport phenomena in the Burnett regime [20, 21]. The crucial question, however, whether the predicted effect can be expected to be of measurable size cannot be answered by such an approach.

In this note, results of a semi-microscopic theory are presented which allow an estimate of the size of

Reprint requests to Prof. Dr. S. Hess, Institut für Theoretische Physik, TU Berlin, Hardenbergstr. 4-5, D-1000 Berlin 12 the heat flow birefringence in liquids and liquid crystals. More specifically, an equation for the torque exerted on a nonspherical particle by the second spatial derivative $\nabla \nabla T$ of the temperature field $T$ is formulated which involves a yet unknown coupling coefficient $K$. Insertion of this torque into a generalized Fokker-Planck equation for the orientation distribution function yields - in analogy to flow birefringence $[9,22]-$ a nonequilibrium alignment causing a birefringence whose magnitude depends on $\nabla \nabla T$ and on the above mentioned $K$. Next, $K$ is calculated for two distinct mechanisms. Firstly, the torque due to a spatial variation of the pressure caused by the inhomogeneous temperature in a system with constant volume (density) is considered. Secondly, for a system at constant pressure, the relevant torque is inferred from the antisymmetric part of the pressure tensor as evaluated from a generalized Enskog-Boltzmann equation for rigid ellipsoids [23] in the limiting case of long needles.

\section{Thermal Torque}

A temperature field $T(\boldsymbol{r})$ is considered which has a vanishing lst spatial derivative, $\nabla T=0$, but which has a nonzero 2nd spatial derivative as (approximately) realized in the experimental set-up described in [7]:

$$
T(\boldsymbol{r})=T_{0}+\delta T
$$

with $T_{0}=T(\boldsymbol{r}=0)$ and

$$
\delta T=\frac{1}{2} \overrightarrow{\boldsymbol{r} r}:(\overparen{\nabla} \boldsymbol{\nabla} T)_{\boldsymbol{r}=0} .
$$

0340-4811 / $85 / 0100-0003 \$ 01.30 / 0$. - Please order a reprint rather than making your own copy. 
In (2), the symbol $\ldots$ refers to the symmetric traceless part of a tensor, e.g.

$$
\vec{a} \boldsymbol{b}=\frac{1}{2}(\boldsymbol{a} \boldsymbol{b}+\boldsymbol{b} \boldsymbol{a})-\frac{1}{3} \boldsymbol{a} \cdot \boldsymbol{b} \boldsymbol{\delta}
$$

for the dyadic constructed from the components of two vectors $\boldsymbol{a}$ and $\boldsymbol{b} ; \boldsymbol{\delta}$ is the unit tensor.

The torque exerted on a nonspherical particle with its figure axis parallel to the unit vector $\boldsymbol{u}$ in such a temperature field is - due to general symmetry arguments - given by

$$
\vartheta_{\mu}=-K \varepsilon_{\mu v i} \overrightarrow{u_{v}} u_{\alpha} \overrightarrow{\nabla_{\alpha}} \nabla_{\lambda} T,
$$

where $K$ is a characteristic coefficient depending on the shape of the particle. In (4), Cartesian components are denoted by Greek subscripts, the summation convention is used for them; $\varepsilon_{\mu \nu \lambda}$ is the antisymmetric third rank isotropic tensor. With the help of the differential operator

$$
\mathscr{f}=\boldsymbol{u} \times \frac{\partial}{\partial \boldsymbol{u}}
$$

the thermal torque (4) can be written as

$$
\vartheta=-\frac{1}{2} K f \vec{u} \boldsymbol{u}: \vec{\nabla} \nabla T .
$$

Notice that $\bar{\nabla} \boldsymbol{\nabla} T \sim \bar{\nabla} \boldsymbol{q}$, where $\boldsymbol{q}$ is the heat flux vector: hence one has

$$
\vartheta=\frac{1}{2} K \lambda^{-1} f \overrightarrow{\boldsymbol{u}} \boldsymbol{u}: \bar{\nabla} \boldsymbol{q}
$$

where $\lambda$ is the heat conductivity of the liquid.

\section{Nonequilibrium Alignment, Heat Flow Birefringence Coefficient}

The torque caused by a shear flow as used in a kinetic theory for the flow birefringence of colloidal solutions [9] and liquid crystals [22] is quite similar to (7); the flow velocity $\boldsymbol{v}$ occurs instead of the heat flow $\boldsymbol{q}$, and a different coupling coefficient has to be used instead of $K \lambda^{-1}$. Due to the close similarity between both problems, the calculation of the alignment in a stationary nonequilibrium situation from a generalized Fokker-Planck equation [22] need not be repeated here. A comparison of the relevant torques shows that the quantity

$$
\varkappa \tau_{a} \bar{\nabla} \boldsymbol{v}
$$

with a characteristic coefficient $\varkappa$ and a relaxation time coefficient $\tau_{a}$ of [22] corresponds to

$$
-\sqrt{\frac{3}{10}} K\left(k_{\mathrm{B}} T\right)^{-1} \nabla \nabla T
$$

of the present problem; $k_{\mathrm{B}}$ is the Boltzmann constant. Hence one infers from [22] that the alignment tensor

$$
\boldsymbol{\alpha}=\sqrt{\frac{15}{2}}\langle\bar{u} \boldsymbol{u}\rangle,
$$

where $\langle\ldots\rangle$ stands for an average evaluated with the orientational distribution function is, for the isotropic phase of a liquid crystal, given by

$$
\mathbf{a}=-\sqrt{\frac{3}{10}} K\left(k_{\mathrm{B}} T\right)^{-1}\left(1-\frac{T^{*}}{T}\right)^{-1} \vec{\nabla} T .
$$

In (11), $T^{*}$ is a pseudo critical temperature somewhat lower than the temperature where the phase transition isotropic-nematic takes place. The factor $\left(1-T^{*} / T\right)^{-1}$ describes a pretransitional increase of the alignment analogous to that one well-known in connection with flow-birefringence [24, 25]. For "normal" molecular liquids and colloidal solutions where collective alignment effects are weak, $1-T^{*} / T$ can be replaced by 1 in (11).

The anisotropic part $\overline{\boldsymbol{\varepsilon}}$ of the dielectric tensor $\boldsymbol{\varepsilon}$ which is responsible for the birefringence is related to the alignment tensor $\mathbf{a}$ by

$$
\overline{\boldsymbol{\varepsilon}}=\varepsilon_{\mathrm{a}}\langle\boldsymbol{u} \boldsymbol{u}\rangle=\varepsilon_{\mathrm{a}} \sqrt{\frac{2}{15}} \mathbf{a}
$$

with the coefficient

$$
\varepsilon_{\mathrm{a}}=\frac{n}{\varepsilon_{0}}\left(\alpha-\alpha_{\perp}\right) .
$$

In (13), $n$ is the number density, $\varepsilon_{0}$ is the absolute dielectric constant of the vacuum, and $\alpha_{\|}, \alpha_{\perp}$ are effective polarizabilities for an electric field vector parallel and perpendicular to the figure axis of a nonspherical particle.

Thus the heat flow birefringence coefficient $\beta_{\lambda}$. defined by the constitutive equation $[1,2]$

$$
\overline{\boldsymbol{\varepsilon}}=-2 \beta_{\lambda}, \boldsymbol{\nabla} \boldsymbol{q}=2 \lambda \beta_{\lambda} \boldsymbol{\nabla \nabla} T
$$

is, according to $(11,12)$ given by

$$
\lambda \beta_{i}=\frac{-1}{10} \varepsilon_{\mathrm{a}} K\left(k_{\mathrm{B}} T\right)^{-1}\left(1-\frac{T^{*}}{T}\right)^{-1} .
$$

Clearly, the magnitude of the coefficient $K$ is of crucial importance for the question whether the heat flow birefringence is of measurable size or not. 


\section{Thermal Torque at Constant Volume}

The torque $\vartheta$ exerted on a particle immersed in a fluid with the pressure tensor $\mathbf{P}$ is given by

$$
\vartheta_{\mu}=-\varepsilon_{\mu \nu \lambda} \int r_{v} P_{\lambda \alpha} n_{\alpha} \mathrm{d} \sigma,
$$

where $\boldsymbol{n}$ is the outer normal of the particle and $\mathrm{d} \sigma$ is the surface element. The point $\boldsymbol{r}=0$ corresponds to the centre of mass of the particle.

Next, the special case of an isotropic, but spatially dependent pressure

$$
\mathbf{P}=P(\boldsymbol{r}) \boldsymbol{\delta}
$$

is considered where the $\boldsymbol{r}$-dependence of $P$ stems from the spatial dependence of $T$ in a system at constant volume:

$$
P(\boldsymbol{r})=P(T(\boldsymbol{r})) \approx P_{0}+\frac{\partial P}{\partial T} \delta T(\boldsymbol{r})
$$

with $P_{0}=P\left(T_{0}\right)$ and $\delta T$ given by (2). It is assumed tacitly that the presence of the particle under consideration - in first approximation - does not affect the temperature field. Insertion of (17) with (18) into (16) shows that $K$ is generally given by

$$
K=\left(\Theta_{\perp}-\Theta_{\|}\right) \frac{\partial P}{\partial T},
$$

where $\Theta_{\|}$and $\Theta_{\perp}$ are the momenta of inertia for density $\varrho=1$ pertaining to rotations around the symmetry axis and the other principal axes through the centre of mass, respectively.

For ellipsoids of revolution with the semiaxes $a=b$ and $c$, (19) yields

$$
K=\frac{4 \pi}{15} a^{2} c^{3}\left(1-\frac{a^{2}}{c^{2}}\right) \frac{\partial P}{\partial T} .
$$

The result for spherocylinders of height $h$ and radius $r_{0}$ is

$$
K=\frac{\pi}{12} r_{0}^{2} h^{3}\left[1+4 \frac{r_{0}}{h}+3\left(\frac{r_{0}}{h}\right)^{2}\right] \frac{\partial P}{\partial T} .
$$

Notice that according to (19), $K$ vanishes for particles with an isotropic inertia tensor, e.g. for $a=c$ in (20) or $h=0$ in (21).

Clearly, the present mechanism generating a torque on a particle does not work if the (scalar) pressure is constant in the presence of a spatially inhomogeneous temperature, yet there are additional mechanisms leading to a thermal torque of the form (4). It seems worth mentioning, however, that insertion of a thermal pressure $\vec{P} \sim \vec{\nabla} \boldsymbol{\nabla} T$ into (16) yields no thermal torque due to $\Delta T=0$ for a stationary heat flux.

\section{Thermal Torque at Constant Pressure for a Fluid of Long Needles, Relation to Flow Birefringence}

In [23], a generalization of the Enskog-Boltzmann equation to rigid ellipsoids has been formulated for the case where the axes of the particles - in first approximation - do not change their directions in a collision. The torque exerted on a particle with a fixed orientation can be inferred from the antisymmetric part of the pressure tensor resulting in a nonequilibrium situation. For the present problem, the analysis was carried out with the help of the moment method and for the limiting case of long thin particles (needles) with length $l$; the result [26] for an isotropic medium (random orientation of the collision partners) is

$$
K=\frac{175}{1536} y_{0} k_{\mathrm{B}} l^{2} \text {. }
$$

Here, $y_{0}<1$ is the volume fraction occupied by the molecules.

It seems worth mentioning that the torque caused by a viscous flow is, in the same approximation, given by

$\vartheta_{\mu}=\frac{7}{18} \sqrt{\frac{\pi}{2}} y_{0} n k_{\mathrm{B}} T c_{0}^{-1} \chi l^{4} \varepsilon_{\mu \nu \lambda} \widetilde{u}_{v} u_{\alpha} \nabla_{\alpha} v_{\lambda}$

with $c_{0}=\sqrt{k_{\mathrm{B}} T / m} ; m$ is the mass of a molecule, and $\chi$ essentially the pair correlation function; cf. Eq. (4) for the thermal torque. The ensuing flow birefringence coefficient $\beta$, defined by

$$
\overrightarrow{\boldsymbol{\varepsilon}}=-2 \beta \widetilde{\nabla v},
$$

is now

$$
\beta=-\frac{7}{180} \sqrt{\frac{\pi}{2}} \varepsilon_{\mathrm{a}} y_{0} n c_{0}^{-1} \chi l^{4}\left(1-\frac{T^{*}}{T}\right)^{-1} .
$$

Comparison of Eqs. $(15,22)$ with $(25)$ yields the following relation between the heat flow and the flow birefringence coefficients:

$$
\lambda \beta_{\lambda}=\frac{75}{128 \sqrt{2 \pi}} c_{0}\left(n T \chi l^{2}\right)^{-1} \beta .
$$




\section{Estimate of the Order of Magnitude of the Effect}

Let $\boldsymbol{e}^{(1)}, \boldsymbol{e}^{(2)}$ be unit vectors parallel to two of the principal axes of the dielectric tensor, in the present case determined by the tensor $\overrightarrow{\nabla \nabla} T$. The difference $\delta v=v_{1}-v_{2}$ between the indices of refraction of light linearly polarized parallel to $\boldsymbol{e}^{(1)}$ and $\boldsymbol{e}^{(2)}$ is given by

$$
v \delta v=\frac{1}{2}\left(\boldsymbol{e}^{(1)} \boldsymbol{e}^{(1)}-\boldsymbol{e}^{(2)} \boldsymbol{e}^{(2)}\right): \bar{\varepsilon}
$$

with the average (isotropic) index of refraction $v$. Thus, due to (15), one has

$$
v \delta v=-\frac{1}{10} \varepsilon_{\mathrm{a}} K k_{\mathrm{B}}^{-1}\left(1-\frac{T^{*}}{T}\right)^{-1} \psi_{12}
$$

with the abbreviation

$$
\psi_{12}=T^{-1}\left(\boldsymbol{e}^{(1)} \boldsymbol{e}^{(1)}-\boldsymbol{e}^{(2)} \boldsymbol{e}^{(2)}\right): \boldsymbol{\nabla} \boldsymbol{\nabla} T .
$$

To estimate the order of magnitude of the difference $\delta v$, it is firstly noted that values

$$
\left|\psi_{12}\right| \approx 10^{4} \mathrm{~m}^{-2}
$$

can be obtained experimentally [7] for the relative second spatial derivative of the temperature field. For an estimate, $v$ and $\varepsilon_{\mathrm{a}}$ can be replaced by 1 in (28), thus

$$
|\delta v| \approx|K| k_{\mathrm{B}}^{-1}\left(1-\frac{T^{*}}{T}\right)^{-1} \cdot 10^{3} \mathrm{~m}^{-2}
$$

for $\psi_{12}$ given by (30).

Next, estimates are given for the coefficient $K$ according to the physical mechanisms discussed in Sect. 3 and 4, respectively.

\section{i) Constant density}

A typical value for $\partial P / \partial T$ occurring in $(19,20$, 21) is $\partial P / \partial T \approx 10^{6} \mathrm{~N}\left(\mathrm{~m}^{2} \mathrm{~K}\right)^{-1}$. With the molecular length parameters chosen to match a substance like

[1] S. Hess, Phys. Lett. 30 A, 239 (1969).

[2] S. Hess, Proc. $7^{\text {th }}$ Internat. Sympos. on Rarefied Gas Dynamics, Pisa 1970; Editrice Tenico Scientifica, ed. D. Dini, Pisa 1971.

[3] S. Hess, Z. Naturforsch. 28 a, 861 (1973).

[4] M. H. Moraal, Physics Reports 17, 225 (1975).

[5] F. Baas, J. N. Breunese, and H. F. P. Knaap, Physica 88 A, 34 (1977).

[6] F. Baas, P. Oudeman, H. F. P. Knaap, and J. J. M. Beenakker, Physica 88 A, 44 (1977).

[7] P. Oudeman, H. F. P. Knaap, and J. J. M. Beenakker, J. Phys. Chem. 86, 1125 (1982).
$\operatorname{MBBA}\left(r_{0} \approx 3 \cdot 10^{-10} \mathrm{~m}, h \approx 1.5 \cdot 10^{-9} \mathrm{~m}\right)$, one finds

$$
K k_{\mathrm{B}}^{-1} \approx 10^{-17} \mathrm{~m}^{2}
$$

and for $\left(1-T^{*} / T\right)^{-1} \approx 10$,

$$
|\delta v| \approx 10^{-13} \text {. }
$$

On the other hand, for ellipsoids with the semiaxes $a \approx 10^{-9} \mathrm{~m}, c \approx 10^{-8} \mathrm{~m}, K k_{\mathrm{B}}^{-1} \approx 10^{-13} \mathrm{~m}^{2}$ and, even with $T^{*} / T \ll 1,|\delta v| \approx 10^{-10}$.

\section{ii) Constant pressure}

In this case, Eq. (22) yields for a substance like MBBA $K k_{\mathrm{B}}^{-1} \approx 10^{-18} \mathrm{~m}^{2}$, thus, for the same enhancement factor $\left(1-T^{*} / T\right)^{-1} \approx 10$ as above, the value $|\delta v| \approx 10^{-14}$ is found. Similarly as above, for fluids of larger (longer) particles, $|\delta v|>10^{-14}$ can be expected on account of (31) with (22). Differences $|\delta v|$ of the indices of refraction as small as $10^{-15}$ can be detected experimentally [5-7]. Thus the heat flow birefringence in liquids should be of measurable size according to the present considerations.

\section{Concluding Remarks}

Two distinct mechanisms have been studied which lead to a heat flow birefringence in liquids. According to the estimates given, the effect is of measurable size. Of particular interest would be experiments on (nematic) liquid crystals in the isotropic phase where a pretransitional increase of the birefringence is expected. Also measurements in dense fluids of larger nonspherical particles (lyotropics) as well as in viscoelastic solutions [27] are desirable.

\section{Acknowledgement}

Financial support by the Deutsche Forschungsgemeinschaft is gratefully acknowledged.

[8] J. C. Maxwell, Proc. Roy. Soc. London (A) 22, 46 (1873); Pogg. Ann. Physik 151, 151 (1874).

[9] A. Peterlin and H. A. Stuart, in Hand- u. Jahrbuch d. Chem. Phys. (Eucken, Wolf, eds.), 8, 113 (1943).

[10] J. V. Champion and G. H. Meeten, Trans. Faraday Soc. 64, 238 (1968).

[11] S. Hess, Springer Tracts Mod. Phys. 54, 136 (1970); Proc. Sympos. "100 years Boltzmann equation", Wien 1972, (E. G. D. Cohen and W. Thirring, eds.), Springer, Wien 1973; Acta Physica Austriaca, Suppl. X, 247 (1973). 
[12] A. G. St. Pierre, W. E. Köhler, and S. Hess, Z. Naturforsch. 27 a, 721 (1972).

[13] F. Baas, Phys. Lett. 36 A, 107 (1971).

[14] G. R. Boyer, B. F. Lamouroux, and B. S. Prade, J. Opt. Soc. Amer. 65, 1319 (1975)

[15] F. Baas, J. N. Breunese, H. F. P. Knaap, and J. J. M. Beenakker, Physica 88 A, 1 (1977).

[16] L. Waldmann, Z. Naturforsch. 12 a, 660 (1957); 13 a, 609 (1958); R. F. Snider, J. Chem. Phys. 32, 1051 (1960).

[17] S. Hess, Z. Naturforsch. 30 a, 728, 1224 (1975); 31 a, 1507 (1976)

[18] I. Pardowitz and S. Hess, Physica 100 A, 540 (1980); S. Hess and I. Pardowitz, Z. Naturforsch. 36 a, 554 (1981).

[19] L. Waldmann and H. Vestner, Physica 80 A, 523 (1975); H. Vestner and L. Waldmann, Physica 86A, 303 (1977).

[20] That Burnett effects usually associated with rarefied gases should also occur in liquids has been demon- strated for the thermal pressure as inferred from the Enskog-Boltzmann equation, cf. G. Schmidt, W. E. Köhler, and S. Hess, Z. Naturforsch. 36 a, 545 (1981).

[21] The diffusion birefringence can also be looked upon as a Burnett type effect, cf. S. Hess, Phys. Lett. 45A, 77 (1973); Physica 74, 277 (1974); J. Halbritter and W. E. Köhler, Physica 76, 224 (1974).

[22] S. Hess, Z. Naturforsch. 31 a, 1034 (1976).

[23] H. Thurn and S. Hess, Z. Naturforsch. 37 a, 660 (1982).

[24] P. G. de Gennes, Phys. Lett. 30 A, 454 (1969).

[25] P. Martinoty, S. Landau, and F. Debeauvais, Phys. Rev. Lett. 27, 1123 (1971).

[26] D. R. Baalss, Transportvorgänge in Flüssigkeiten und Flüssigkristallen aus stark nichtsphärischen Molekülen, Diplomarbeit (unpublished), Erlangen 1982.

[27] H. Thurn, M. Löbl, and H. Hoffmann, J. Phys. Chem. (1984). 